\title{
Residual fragments after percutaneous nephrolithotomy: cost comparison of immediate second look flexible nephroscopy versus expectant management
}

Raman JD, Bagrodia A, Bensalah K, Pearle MS, Lotan Y

Department of Urology, University of Texas Southwestern Medical Center, Dallas, Texas, USA

J Urol. 2010; 183: 188-93

Purpose: We performed a cost comparison of immediate second look flexible nephroscopy vs expectant management for post-percutaneous nephrostolithotomy residual fragments.

Materials and Methods: We used a decision analysis model to compare the cost of managing residual fragments by second look flexible nephroscopy vs observation. Outcomes of residual fragments after percutaneous nephrostolithotomy were determined from institutional experience and published shock wave lithotripsy series. Cost data were obtained from billing records. One-way sensitivity analysis was done to evaluate incurred costs of second look flexible nephroscopy while varying the likelihood of a stone event, the probability of surgery and the cost of surgical intervention. Two-way sensitivity analysis was done to assess the model across a range of scenarios.

Results: Based on data in the literature and our institutional experience $40 \%$ of patients with residual fragments $4 \mathrm{~mm}$ or less had a stone event, of whom $57 \%$ required surgical intervention. Based on these estimates the average cost of expectant management for a residual fragment $4 \mathrm{~mm}$ or less vs greater than 4 was $\$ 1,743$ vs $\$ 4,674$. The average incremental cost of second look flexible nephroscopy at our institution was $\$ 2,475$. Two-way sensitivity analysis showed that varying assumptions dramatically altered conclusions about the cost benefit of second look flexible nephroscopy.

Conclusions: Our model suggests that second look flexible nephroscopy is not cost advantageous in all patients with post-percutaneous nephrostolithotomy residual fragments. Cost benefit analysis is significantly impacted by the likelihood of a stone related event, the need for surgical intervention and surgical costs. Compared to an observational strategy second look flexible nephroscopy incurs lower costs for greater than $4 \mathrm{~mm}$ but not for 4 $\mathrm{mm}$ or less residual fragments.

\section{Editorial Comment}

The authors have conducted a critical appraisal of their protocol for CT scan imaging on postoperative day 1 followed by second look nephroscopy. The current study confirms that second-look flexible nephroscopy (SLFN) is not warranted for residual stone fragments smaller than $2 \mathrm{~mm}$, but is a good approach for fragments larger than 4mm. A "grey zone" exists for stones 3 and $4 \mathrm{~mm}$ in size; though cost of observation vs. SLFN is equivalent in this group, $75 \%$ of those observed will experience a stone related event and as such, these patients may benefit clinically from a SLFN. The authors were limited in their ability to build a decision model based on data from patients undergoing PCNL due to the scarcity of studies reporting long-term outcomes with residual fragments in this setting. The use of SWL literature to build the decision model may be limited by the difference in initial stone burden between the two patient groups. Patients with a smaller stone burden (SWL) may be more likely to undergo less invasive secondary procedures for residual fragments - indeed the distribution of secondary procedures used in the metanalysis strongly favored SWL (77\%); one would anticipate a higher use of endoscopic procedures post-PCNL. The decision to select SLFN vs. URS or SWL is often determined by the quality of the initial percutaneous renal access and the location of the RF in relation to the access. With the stone size criteria established, one must now re-evaluate the need for postoperative CT scan imaging - indeed 
intraoperative fluoroscopy with magnification in conjunction with endoscopic evaluation may be sufficient to identify those fragments that really matter.

Dr. Manoj Monga

Professor, Department of Urology

University of Minnesota

Edina, Minnesota, USA

E-mail:endourol@yahoo.com 